
\title{
3 Research Square \\ The Effects of Pulsed Electric Field On The Interaction Between SARS-Cov-2 And Human ACE2: Y505 Is A Key Target
}

\section{Ming-hui Ji}

Shanghai University of Electric Power

Jia-hao Xu

Shanghai University of Electric Power

Sha-sha Yuan

Shanghai University of Electric Power

Ya-wen Liu

Shanghai University of Electric Power

\section{Xin-yi Xing}

Shanghai University of Electric Power

Chao Jiang

Shanghai University of Electric Power

Liang Xue ( $\nabla$ xueliangokay@gmail.com )

Shanghai University of Electric Power https://orcid.org/0000-0003-3233-5983

Chuan-kai Yang

State Grid Shaanxi Electric Power Research Institute

Feng-hong Chu

Shanghai University of Electric Power

You-hua Jiang

Shanghai University of Electric Power

\section{Research Article}

Keywords: COVID-19, Pulsed electric field, MD simulation, RBD, ACE2.

Posted Date: January 4th, 2022

DOI: https://doi.org/10.21203/rs.3.rs-1059300/v1

License: (c) (1) This work is licensed under a Creative Commons Attribution 4.0 International License.

Read Full License 


\section{Abstract}

A novel coronavirus has rapidly spread to almost every country in the world, causing over 233 million confirmed cases of coronavirus disease 2019 (COVID-19) and over 209,761,242 deaths by late September 2021. Binding the receptor binding domain (RBD) to the host cell surface receptor protein, angiotensin converter enzyme (ACE2), is a key step in virus infection. In this study, we applied a pulsed electric field to the RBD/ACE2 complex based on molecular dynamics simulation and demonstrated that the electric field affects the structure and binding affinity of the complex. Additionally, residue Y505 is the crucial medium for the effects of electric field on the complex. Overall, these results may help apply an external electric field to virus suppression.

\section{Introduction}

The epidemic of Coronavirus disease 2019 (COVID-19) caused by severe acute respiratory syndrome coronavirus 2 (SARS-CoV-2) presents a tremendous threat to global public health. SARS-CoV-2 is a positive-sense single-stranded RNA genome [1]. The virus contains four structural proteins, including spike (S) protein, membrane (M) protein, envelope (E) protein and nucleocapsid $(\mathrm{N})$ protein [2]. Among them, $\mathrm{S}$ protein is a virus shell component, which induces the virus to bind to the host cell receptor to generate antibodies or cellular immune response $[3,4]$. Specifically, protein spike (S) contains two subunits: S1 and S2. The spherical S1 and the rod-shaped S2 are combined with each other through intermolecular forces, but their functions are different. $\mathrm{S} 1$ is responsible for binding to receptors on the surface of host cells. Then S2 fixes the entire S protein on the membrane, mediating the fusion of the virus envelope with the host cell membrane [5]. The binding of the receptor binding domain (RBD) on the S1 subunit to human angiotensin converting enzyme 2 (ACE2) contributes to the recognition and attachment of the virus to the host cell [3]. Specifically, residues D38, K31, E37, K353, and Y41 on ACE2 and Q498, T500, and R403 on the RBD contribute to the stability of the relevant protein-protein interface [6]. The amino acid exchanges S477G and S477N strengthen the binding of the SARS-COV-2 spike with the ACE2 [7]. In addition to previously reported residues, it was found that residue F486 located in L3 plays a crucial role in the dynamic stability of the complex by a $\pi$-stacking interaction with ACE2 [8]. Besides, the SARS-CoV-2 polybasic cleavage sites via electrostatic interactions and hydration enhance the RBD-ACE2 binding affinity, although they distributed approximately $10 \mathrm{~nm}$ away from the RBD. [9]. From the perspective of interaction, networks of hydrogen-bonding and hydrophobic interactions enhance receptor binding of SARS-CoV-2 [10]. Besides, bridge water molecules play a significant role in stabilizing the protein-protein binary complex [11].

In this study, we applied a certain pulsed electric field to the RBD-ACE2 complex. This research revealed the molecular mechanism of interactions changes in time and might provide a new idea for inhibiting the binding of the SARS-COV-2 spike to ACE2.

\section{Methods}


The complete model was obtained in CHARMM GUI (PDB ID 7DMU) [12]. The complex was initially solvated with 66140 TIP3P water molecules [13] and contained $187 \mathrm{Cl}$ - and $214 \mathrm{~K}+$ (corresponding to a physiological concentration of $0.15 \mathrm{M}$ ). Simulations and analyses of the system were performed using the GROMACS package [14] with CHARMM36 force field [15]. A periodic boundary condition was applied and the temperature was coupled to $310.15 \mathrm{~K}$ with the V-rescale algorithm [16] and the coupling constants were $1.0 \mathrm{ps}$. The pressure was coupled to 1 bar using the Berendsen method [17] and the coupling constants were 5.0 ps. The LINCS algorithm [18] was applied to constrain the covalent bonds with $\mathrm{H}$-atoms. The time step was $2.0 \mathrm{fs}$. The Lennard-Jones effect was truncated at $1.2 \mathrm{~nm}$ and translated from $0.9 \mathrm{~nm}$ to $1.2 \mathrm{~nm}$. The particle mesh Ewald (PME) method was used [19] with a cutoff radius of $1.2 \mathrm{~nm}$. Binding free energy $(\Delta G)$ was calculated using the gmx-MMPBSA method [20]. VMD was used for visualization of results and generation of figures [21].

\section{Results}

\subsection{Structural analysis}

RMSD for the RBD-ACE2 complex was found to be $0.4 \mathrm{~nm}$ as shown in Fig. 1a. The RMSD plot indicates that the complex reached equilibrium at $90 \mathrm{~ns}$. The RMSD of complex backbone was fluctuant under the pulsed electric field of $0.05 \mathrm{v} / \mathrm{nm}$ and gradually increased under the field of $0.075 \mathrm{v} / \mathrm{nm}$ as Fig. $1 \mathrm{~b}$ shows. It can be observed from RMSF plots shown in Fig. 1.c-d that the fluctuation of most of the residues of complex is not influenced by the presence of the external electric field. Besides, the maximum RMSF difference of RBD residues is much less than ACE2 as shown in Fig. 1e-f, which indicates that the RBD has higher stability. Fig. $1 \mathrm{~g}$ and $\mathrm{h}$ show that the complex is polarized by the electric field and tends to the direction of the electric field, which might contribute to the changes of RMSD when applied to an electric field. In detail, the ACE2 residues D136-E140 or N338 have larger fluctuations than other residues not containing the terminal residues that fluctuate greatly. These residues still show high fluctuation under an electric field. Interestingly, few residues like L148 and N149 could be affected by the electric field which show higher fluctuation than that under no electric field. Overall, the fluctuation of most of the residues would not be affected by the electric field. Therefore, the increase or fluctuation in RMSD under the electric field is mainly attributed to the movement of the protein backbone instead of the fluctuation of residues. In other words, the electric field only affects the backbone structure of complex but has little effect on the movement of individual residues, which reflects the stability of the binding region of RBD and ACE2.

\subsection{Interaction between RBD and ACE2 without electric field}

Within a distance cutoff of $4 \AA$, the RBD residues (R403, K417, Y449, Y453, K458, F456, G476, E484, N487, F490, Q493, Q498, G502, Y505) contact with the ACE2 residues (S19, E23, Q24, F28, D30, N31, K35, E37, D38, Q42, L45, F79, Y83, N330, K353, D355, R357, R393). The hydrogen bonds are calculated based on the cut-off criteria: (a) donor -acceptor distance $\leq 0.35 \mathrm{~nm}$ and (b) donor $-\mathrm{H}$ - acceptor bond angle $\leq 120^{\circ}$. Salt bridges are defined using a $4 \AA$ cutoff between carboxyl carbon and amine/guanidine 
nitrogen. As Table 1 shows, hydrogen bonds are formed between 7 pairs of residues and one pair of residues form the salt bridge. The occupancy indicates high binding stability of complex which might contribute to high infinity of RBD with ACE2. Fig. 2c shows some residues bind through the hydrogenbonded network formed with water molecules, which we called water bridges. Other residues bind through hydrophobic interaction. In short, these interaction types cause RBD/ACE2 to bind tightly.

\section{Table 1}

Hydrogen bonds and Salt bridges between COV2 and ACE2 that Persist for $>30 \%$.

\begin{tabular}{|llll|}
\hline & RBD & ACE2 & Occupancy (\%) \\
\hline 1 & Q493 & K35 & 75.0 \\
\hline 2 & N487 & Y83 & 94.5 \\
\hline 3 & K417 & D30 & 95.9 \\
\hline 4 & N487 & Q24 & 30.4 \\
\hline 5 & T500 & D355 & 97.7 \\
\hline 6 & G502 & K353 & 94.1 \\
\hline 7 & Y505 & E37 & 89.1 \\
\hline 8 & K417 & D30 & 99.2 \\
\hline \multicolumn{4}{|l}{ Salt bridge is shown as bold. } \\
\hline
\end{tabular}

\subsection{Interaction between RBD and ACE2 with electric field}

As shown in Fig. 3a-c, the number of hydrogen bonds would decrease at the peak of the electric field and the average number of hydrogen bonds formed by Y $505-\mathrm{E} 37$ decreased from 0.90 to 0.59 . The occupancy of hydrogen bonds formed by Y $505-E 37$ decreased as the intensity of electric field is increased. However, the occupancy of salt bridge is close to $99 \%$ regardless of the intensity of electric field. It indicates that the salt bridge has higher stability than hydrogen bonds for the complex. Additionally, the number of bridging water molecules at the contact interface of the RBD and ACE2 is barely affected by the electric field and the average in each case is close to 8 as shown in Fig. 3d-f. Besides, the electric field has little effect on the minimum distance between the RBD and ACE2 as shown in Fig. 3g-l, which indicates that the structure of the contact area between RBD and ACE2 could resist the interference of external electric field. However, the residue Y 505 as a notable target provides a chance for the electric field to decrease the binding affinity of complex through hydrogen bonds.

\subsection{Binding free energy}


1000 frames of $10 \mathrm{~ns}$ trajectory were used for the calculation of binding free energy. The binding free energy of a ligand-receptor complex can be calculated as: $\Delta \mathrm{Gbind}=\Delta \mathrm{H}-\mathrm{T} \Delta \mathrm{S}$. Here, only effective energy $(\Delta \mathrm{H})$ was calculated, neglecting the entropy contribution(-T $\Delta S$ ). It can be observed that the binding free energy starts to decrease at 500 frames which reaches the peak of pulsed electric field as shown in Fig. 4. The decomposition analysis shown in Fig. $4 a$ and $\mathrm{c}$ indicates that the binding affinity of residue Y 505 reduced from $4.6 \mathrm{kcal} / \mathrm{mol}$ to $2.2 \mathrm{kcal} / \mathrm{mol}$. Besides, the affinity of $Y 505$ under the field of $0.075 \mathrm{v} / \mathrm{nm}$ decreased significantly over most of the time contrary to the field of $0.05 \mathrm{v} / \mathrm{nm}$ as shown in Fig. $4 \mathrm{~b}$ and d. Hence, residue $Y 505$ is easily affected by external electric field contrary to other residues. In combination with section 3.3, the binding affinity and the number of hydrogen bonds for Y 505 decrease as the electric field is increased. It indicates that Y505 might be the hot pot that could be applied to the suppression of binding between RBD and ACE2.

\section{Discussion}

In this work, we performed MD simulations to unveil the detailed molecular mechanism for the effects of pulsed electric field on the receptor binding of nCOV-2019. The binding affinity of complex is 57.96 $\mathrm{kcal} / \mathrm{mol}$, whereas the complex under the field of $0.075 \mathrm{v} / \mathrm{nm}$ has a lower binding affinity of 7.67 $\mathrm{kcal} / \mathrm{mol}$. Hydrogen bonds, salt bridges, water bridges and hydrophobic contacts [9-11] enhance the binding affinity of RBD-ACE2. However, only hydrogen binding interaction can be affected by the electric field. Moreover, the hydrogen-bonded network formed by most of the residues except Y505 and E37 could bare the interference of electric field. The binding affinity increases shown in Fig. 4 as the pulse the electric field disappeared, which indicates that the stability of RBD-ACE2 could not be destroyed completely. Hence, the destruction of binding affinity should focus on the peak time of pulsed electric field.

\section{Conclusion}

In the research, we study the effects of external electric field on the RBD-ACE2 complex with molecular dynamics methods for the first time. We proposed that the structure and binding affinity of complex can be affected by the external electric field. Additionally, the residue Y 505 of RBD plays an important role in the binding affinity of RBD/ACE2. In detail, the electric field decreases the binding affinity by destroying the hydrogen-bonded network between Y505 and E37. Our findings may provide a theoretical basis for the application of electric field to virus suppression.

\section{Declarations}

Funding This work was supported by National Natural Science Foundation of China (62105196) and Shanghai Sailing Program (17YF1407000).

Conflicts of interest All the authors declare no conflicts of interest. 
Availability of data and material N/A

\section{Code availability N/A}

\section{Ethics approval N/A}

Author contribution Ming-hui Ji: Software, Validation, Investigation, Writing - original draft. Jia-hao Xu: Writing - review \& editing. Sha-sha Yuan: Data analysis. Ya-wen Liu: Image processing. Xing-yi Xing: Review \& editing. Chao Jiang: Editing, Supervision. Liang Xue: Research conception, Data curation, Writing - review \& editing, Supervision. Chuan-kai Yang: Editing. Feng-hong Chu: Editing. You-hua Jiang: Editing.

\section{References}

1. Lai MM, Cavanagh D (1997) The molecular biology of coronaviruses. Adv Virus Res 48:1-100

2. Su S, Wong G, Shi W et al (2016) Epidemiology, genetic recombination and pathogenesis of coronaviruses. Trends Microbiol 24(6):490-502

3. Gui M, Song W, Zhou H, Xu J, Chen S, Xiang Y, Wang X (2017) Cryo-electron microscopy structures of the SARS-CoV spike glycoprotein reveal a prerequisite conformational state for receptor binding. Cell Res 27(1):119-129

4. Kirchdoerfer RN, Cottrell CA, Wang N et al (2016) Pre-fusion structure of a human coronavirus spike protein. Nature 531(7592):118-121

5. Li F (2016) Structure, function and evolution of coronavirus spike proteins. Annu Rev Virol 3(1):237261

6. Laurini E, Marson D, Aulic S et al (2020) Computational Alanine Scanning and Structural Analysis of the SARS-CoV-2 Spike Protein/Angiotensin-Converting Enzyme 2 Complex. ACS Nano 14(9):1182111830

7. Singh A, Steinkellner G, Köchl K et al (2021) Serine 477 plays a crucial role in the interaction of the SARS-CoV-2 spike protein with the human receptor ACE2. Sci Rep 11:4320

8. Mahdi Ghorbani BR, Brooks, Klauda JB. Critical Sequence Hotspots for Binding of Novel Coronavirus to Angiotensin Converter Enzyme as Evaluated by Molecular Simulations. The Journal of Physical Chemistry B 2020124 (45), 10034-10047

9. Baofu Qiao and Monica Olvera de la Cruz (2020) Enhanced Binding of SARS-CoV-2 Spike Protein to Receptor by Distal Polybasic Cleavage Sites. ACS Nano 14(8):10616-10623

10. Yingjie Wang M, Liu J, Gao. Enhanced receptor binding of SARS-CoV-2 through networks of hydrogen-bonding and hydrophobic interactions. Proceedings of the National Academy of Sciences Jun 2020, 117 (25) 13967-13974

11. Malik A, Prahlad D et al. Interfacial Water Molecules Make RBD of SPIKE Protein and Human ACE2 to Stick Together. bioRxiv 2020.152892 
12. Jo S, Kim T, lyer VG, Im W (2008) J Comput Chem 29:1859-1865

13. Jorgensen WL, Chandrasekhar J, Madura JD (1983) Comparison of simple potential functions for simulating liquid water. J Chem Phys 79:926

14. Abraham M, Murtola T, Schulz R et al (2015) GROMACS: high performance molecular simulations through multi-level parallelism from laptops to supercomputers. SoftwareX 1-2:19-25

15. Pastor RW (2011) and A. D. MacKerell,Development of the CHARMM Force Field for Lipids. The Journal of Physical Chemistry Letters 2(13):1526-1532

16. Bussi G, Donadio D, Parrinello M (2007) Canonical sampling through velocity rescaling. J Chem Phys 126:14101

17. Berendsen HJ, Postma JV, van Gunsteren WF, DiNola AR, Haak JR (1984) Molecular dynamics with coupling to an external bath. J Chem Phys 81(8):3684-3690

18. Hess B (2008) P-LINCS: a parallel linear constraint solver for molecular simulation. J Chem Theory Comput 4:116-122

19. Essmann U, Perera L, Berkowitz ML et al (1995) A smooth particle mesh Ewald method. J Chem Phys 103:8577

20. Mario S. Valdés Tresanco ME, Valdes-Tresanco PA, Valiente, \& Ernesto Moreno Frías. gmx_MMPBSA (Version v1.4.3). Zenodo

21. Humphrey W, Dalke A, Schulten K (1996) VMD: Visual molecular dynamics. J Mol Graph 14:33-38

\section{Figures}



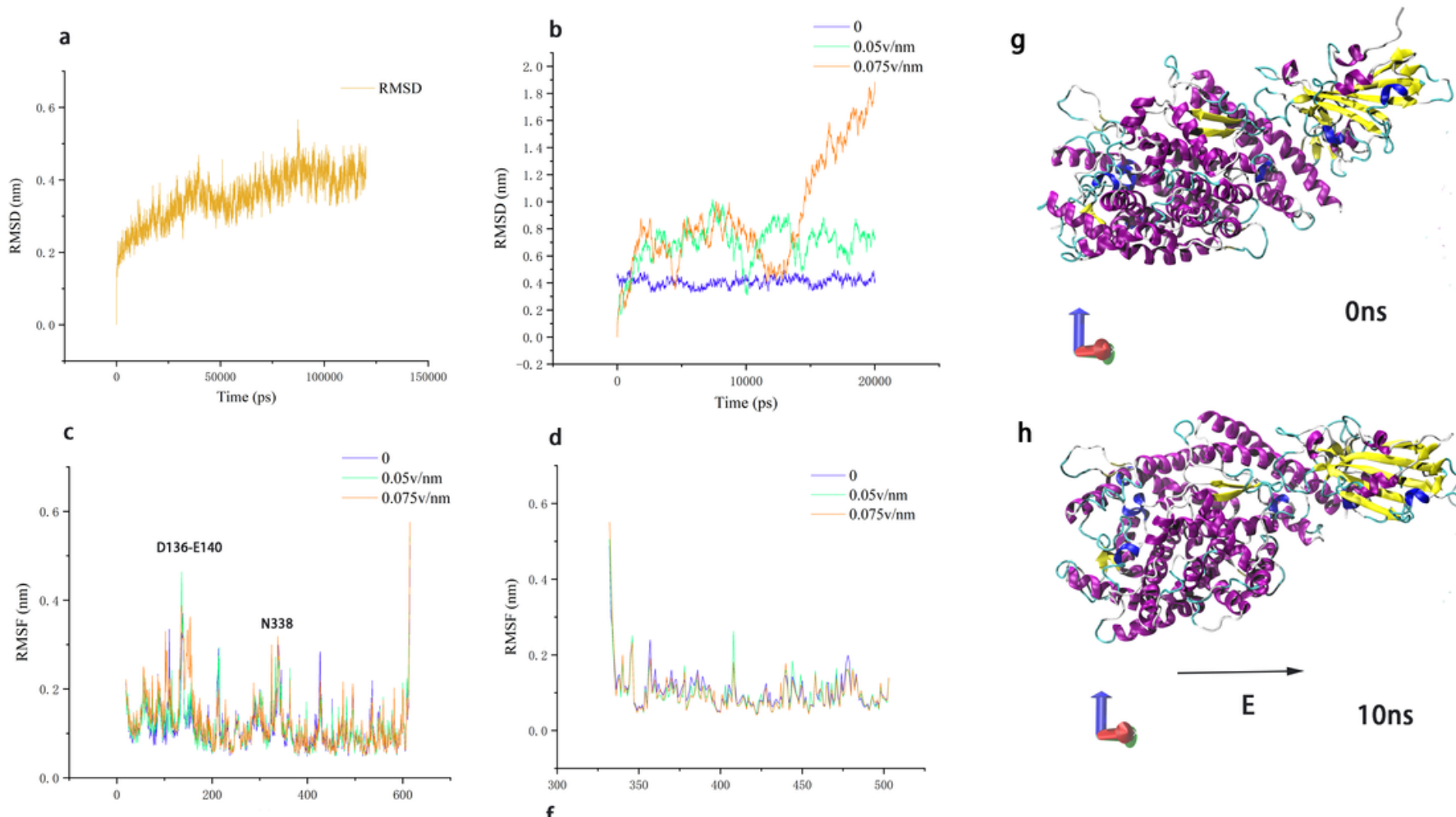

h
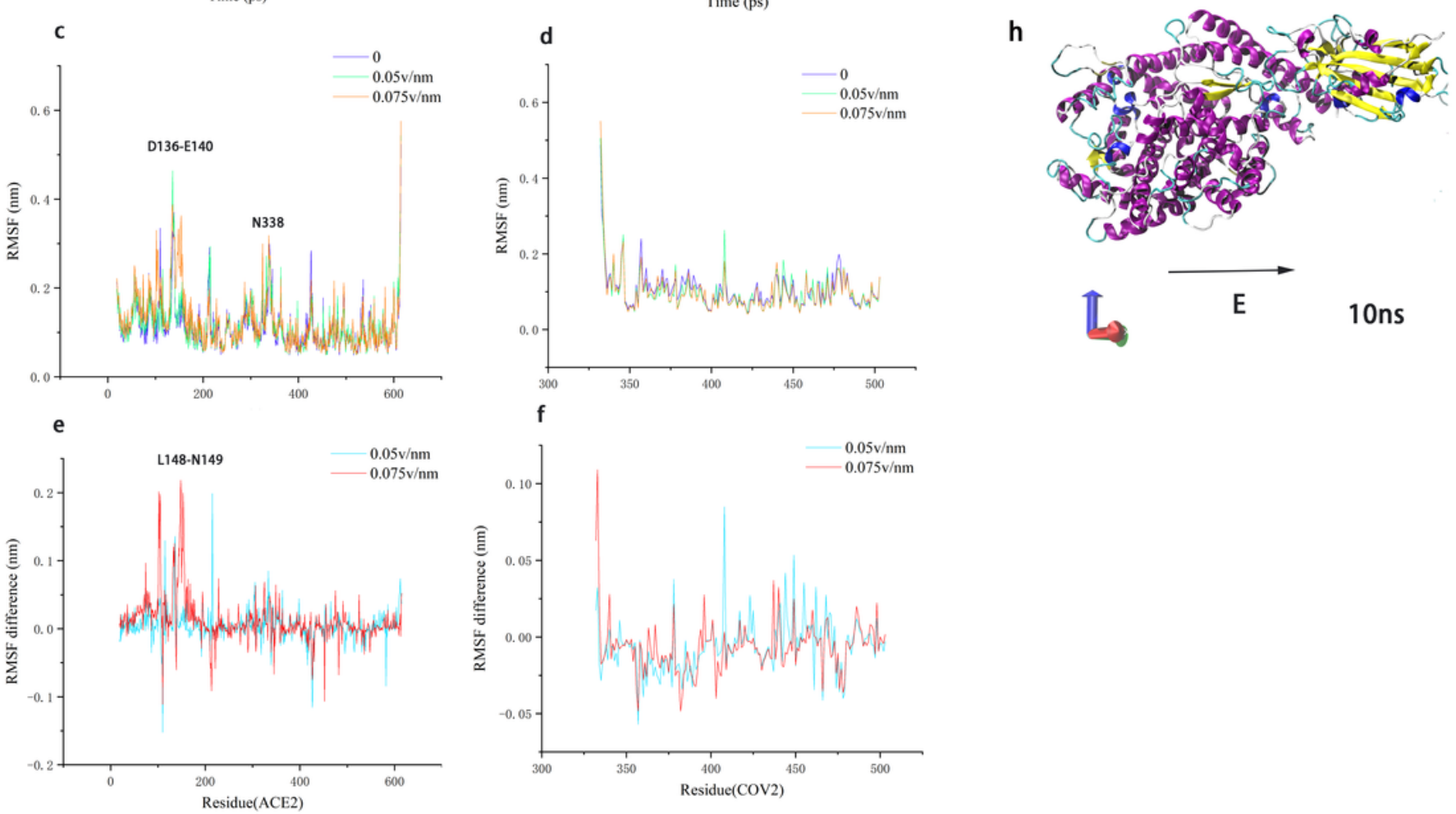

\section{Figure 1}

RMSD and RMSF plots for the RBD/ACE2 complex. a represents RMSD plots for the entire simulation. $b$ represents RMSD plots for the complex backbone under different electric fields. $\mathrm{c}$ and e represent RMSF plots for the residues of ACE2 under different electric fields. $d$ and $f$ represent RMSF plots for the residues of RBD. $g$ and $h$ represent the structure changes of complex under the electric field. 
a

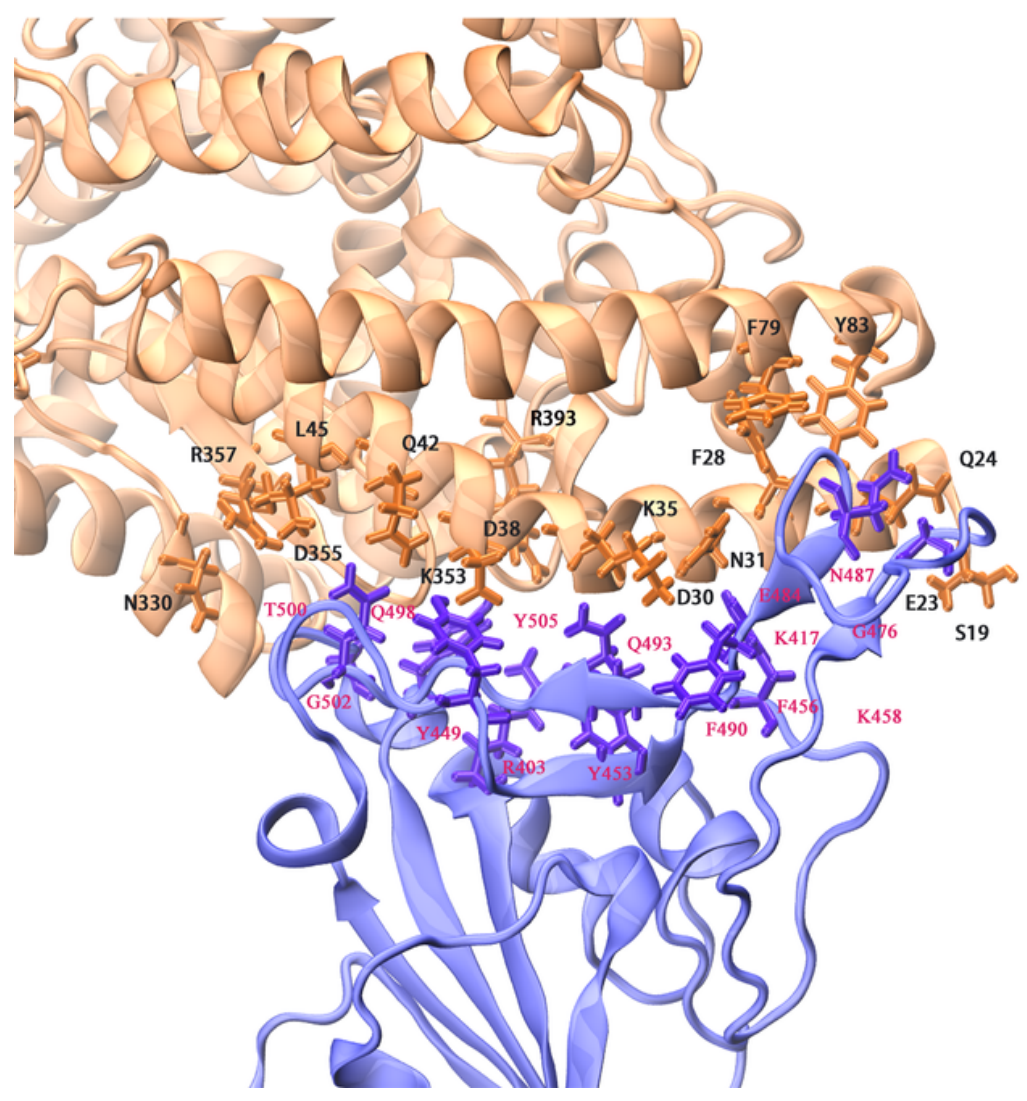

b
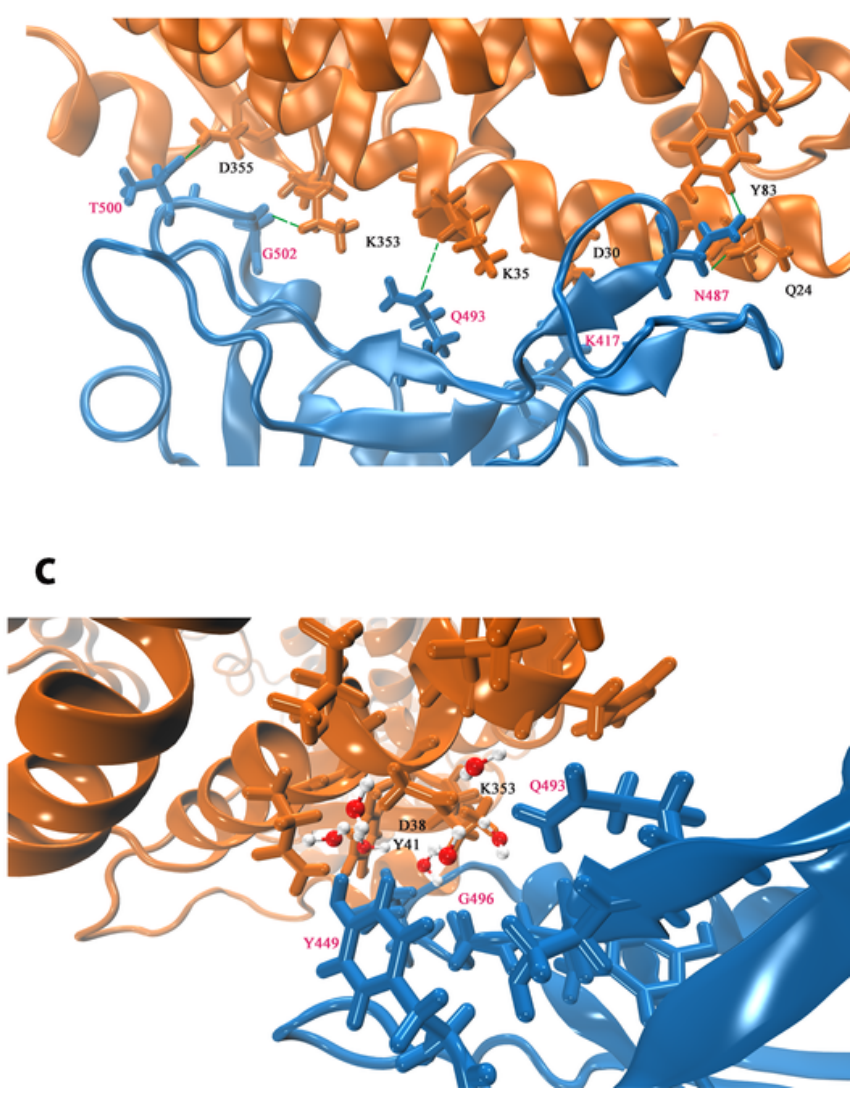

Figure 2

Interaction between RBD and ACE2. a represents residues at the touching interface between RBD and ACE2. $b$ represents the hydrogen bonds of complex and the hydrogen bonds are shown by green dashed lines. c shows some water molecules which form hydrogen bonds with residues from RBD and ACE2. 

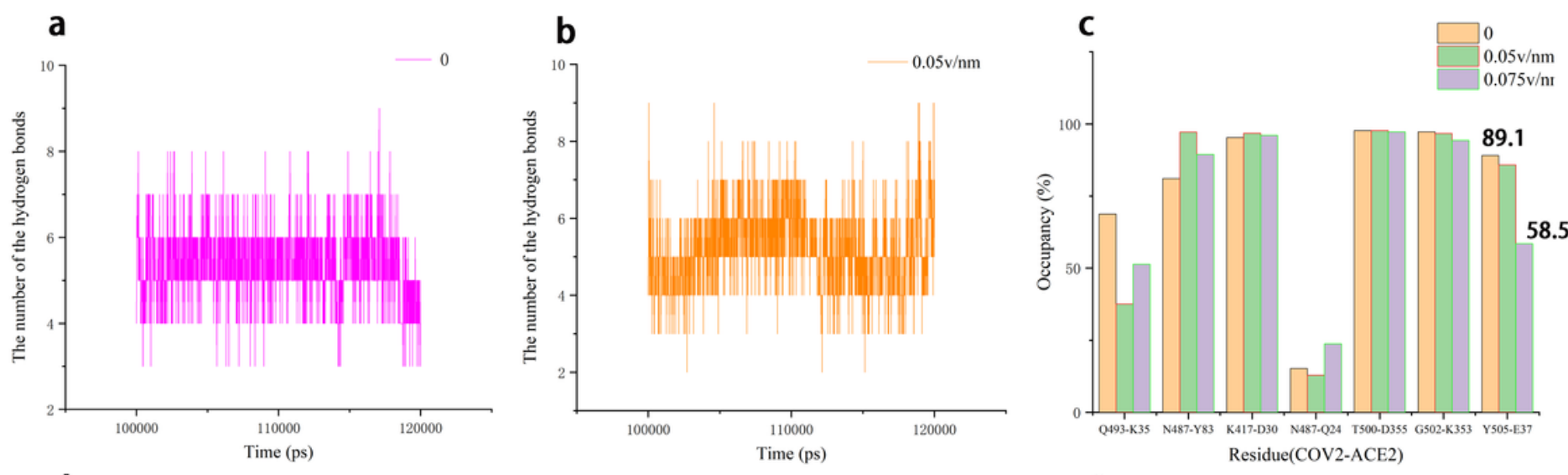

d

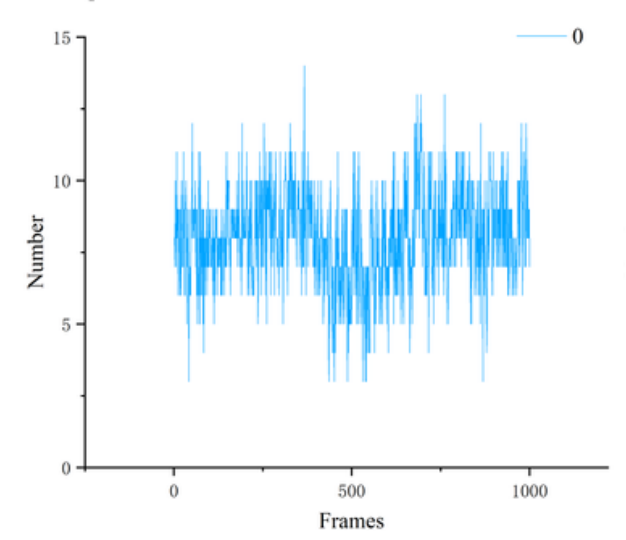

e
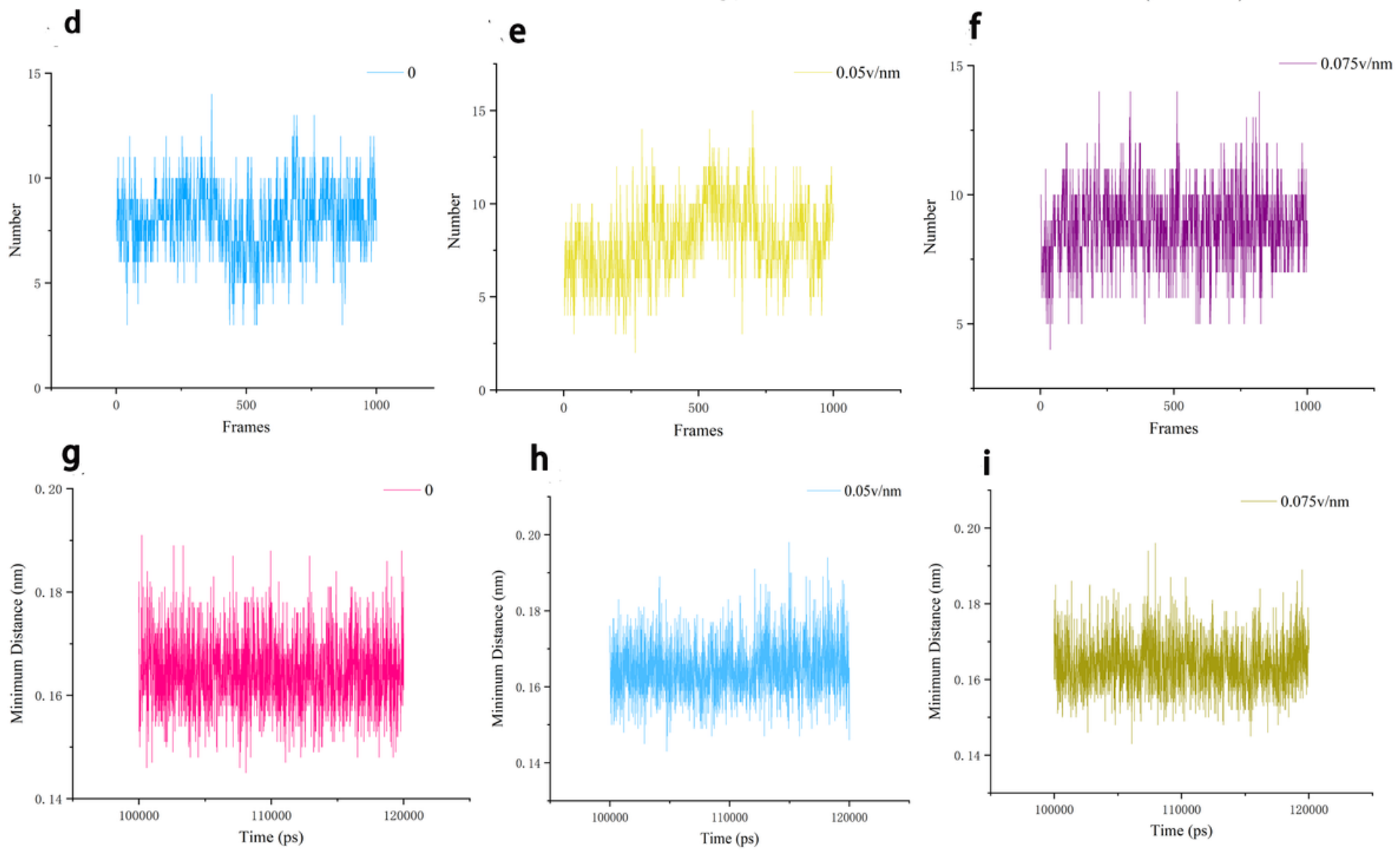

Figure 3

Comparison of interaction between RBD and ACE2 under the electric field with the interaction under no electric field. a represents the number of hydrogen bonds of RBD/ACE2 under no electric field. $b$ represents the number of hydrogen bonds under the field of $0.05 \mathrm{v} / \mathrm{nm}$. $\mathrm{c}$ represents the changes of occupancy under different electric fields. $d$ and $f$ represent the changes in the number of water molecules of water bridges. $g$ and $f$ represent the changes in the minimum distance between RBD and ACE2. 

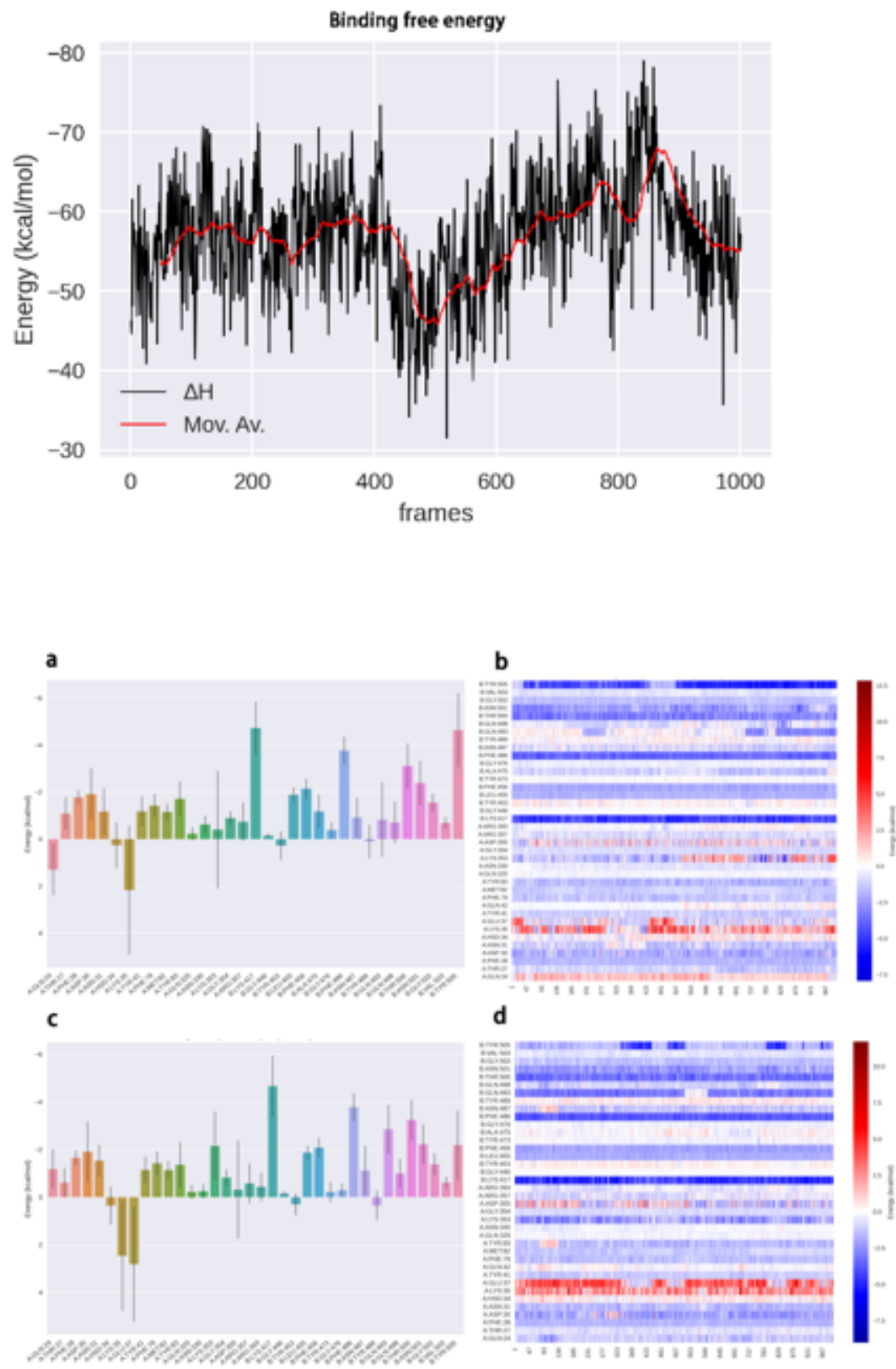

Figure 4

Binding free energy and decomposition analysis of the complex. a represents the total binding free energy decomposition per residue under the field of $0.05 \mathrm{v} / \mathrm{nm}$. b represents the changes of binding energy over time under $0.05 \mathrm{v} / \mathrm{nm}$. $c$ and $d$ are under the field of $0.075 \mathrm{v} / \mathrm{nm}$.

\section{Supplementary Files}


This is a list of supplementary files associated with this preprint. Click to download.

- GraphicalAbstract.bmp

- movie.gif 